\title{
Nicht Kammer sondern Patient als Schlüssel zur Anerkennung der Pflege
}

\author{
Heinz Lohmann
}

Online publiziert: 30 . November 2011

(C) Springer-Verlag 2011

Der postindustrielle Umbruch der gesellschaftlichen Institutionen und Unternehmen hat inzwischen auch die Gesundheitswirtschaft erreicht.

Die auseinander gehende Schere zwischen steigender Nachfrage nach Gesundheits- und Sozialleistungen vor dem Hintergrund der Innovationen moderner Medizin und der Demographie einerseits sowie begrenzten finanziellen Mitteln aus dem Sozialtransfer andererseits hat die gesundheitsund sozialpolitische Debatte der letzten 20 Jahre bestimmt. Das Thema Kosten war und ist der Treiber des Wandels in der Gesundheitswirtschaft.

Innovative Medizin gepaart mit Produktivitätsdruck fördert die Spezialisierung in den Arbeitsprozessen. Die Pflege hat deshalb in den letzten 60 Jahren eine Fülle von spezifischen Berufsbildern durch Ausgliederung ,erzeugt“. Diese neuen Akteure drängen den Aktionsradius der Pflegekräfte immer mehr zurück und lassen deren Aufgaben zur „Restgröße" werden.

Eine ganze Reihe von Pflegekräften und insbesondere viele Funktionäre erwarten von einer Pflegekammer vor allem die Aufwertung ihres Berufsstandes. Sie fühlen sich nicht richtig wertgeschätzt und hoffen, mit einer solchen Institution zum Status der Ärzte aufschließen zu können. Auch möchten sie der Position der Pflege in der öffentlichen Diskussion mehr Gewicht verleihen. Pflegekammern wären aber nur der verlängerte Arm der staatlichen Bürokratie. Innovative Pflegekräfte würden Kammern eher als Bremse denn als Motor erleben.
Die Patientensouveränität auf der Basis zunehmender Transparenz wächst und wird neben der Forderung nach Hebung der Produktivitätsreserven zum zweiten großen Treiber der Wandels in der Gesundheitswirtschaft. Deshalb muss die Devise ,,schneller Arbeiten“ durch ,,anders Arbeiten" ersetzt werden.

In einer Situation, in der die Patientensouveränität aufgrund zunehmender Transparenz steigt, kommt es für Gesundheitsunternehmen mehr und mehr darauf an, die Nachfrageerwartungen zu erfüllen. Pflegekräfte wissen genau, was Patienten und Klienten wollen. Wegen der großen Nähe zu ihnen sind sie die Serviceexperten. Ihr Wissen und ihre Erfahrung werden deshalb mit dem sich verschärfenden Wettbewerb der Gesundheits- und Sozialanbieter immer wertvoller.

In einer sich wandelnden Gesundheits- und Sozialwirtschaft muss Pflege ihre Berufsbilder deutlich konzentrieren und klar strukturieren. Gestaffelte Bildungsangebote als Voraussetzung für Professionalität sind unabdingbar. Nur wer so positioniert einen wertvollen Beitrag zur Lösung künftiger Herausforderungen leisten kann, ist gesellschaftlicher Anerkennung sicher. Pflegende können in der wachsenden Gesundheits- und Sozialwirtschaft ihre Position stärken, wenn sie sich aktiv in die Diskussionen um die Modernisierung der Branche einmischen.

H. Lohmann $(\bowtie)$

LOHMANN konzept GmbH,

Hamburg, Deutschland 